\title{
Biliary Tract Obstruction Due to Sarcoidosis
}

G. C. Sotiropoulos', U. Dahmen',

0. Dirsch ${ }^{2}$, G. Antoch ${ }^{3}$, L. Freudenberg ${ }^{4}$,

E. I. Brokalaki', U. Treichel', A. Paul',

C. E. Broelsch ${ }^{1}$

${ }^{1}$ Department of General Surgery and Transplantation, University Hospital Essen, Essen, Germany

${ }^{2}$ Institute of Pathology, University Hospital Essen, Essen, Germany

${ }^{3}$ Department of Diagnostic and Interventional Radiology, University Hospital Essen, Essen, Germany

${ }^{4}$ Department of Nuclear Medicine, University Hospital Essen, Essen, Germany

${ }^{5}$ Department of Gastroenterology and Hepatology, University Hospital Essen, Essen, Germany

\section{Corresponding Author}

\section{G. C. Sotiropoulos, M.D.}

Department of General Surgery and Transplantation

University Hospital Essen

Hufelandstraße 55

45122 Essen

Germany

Fax: +49-201-7231113

E-mail: georgios.sotiropoulos@uniessen.de
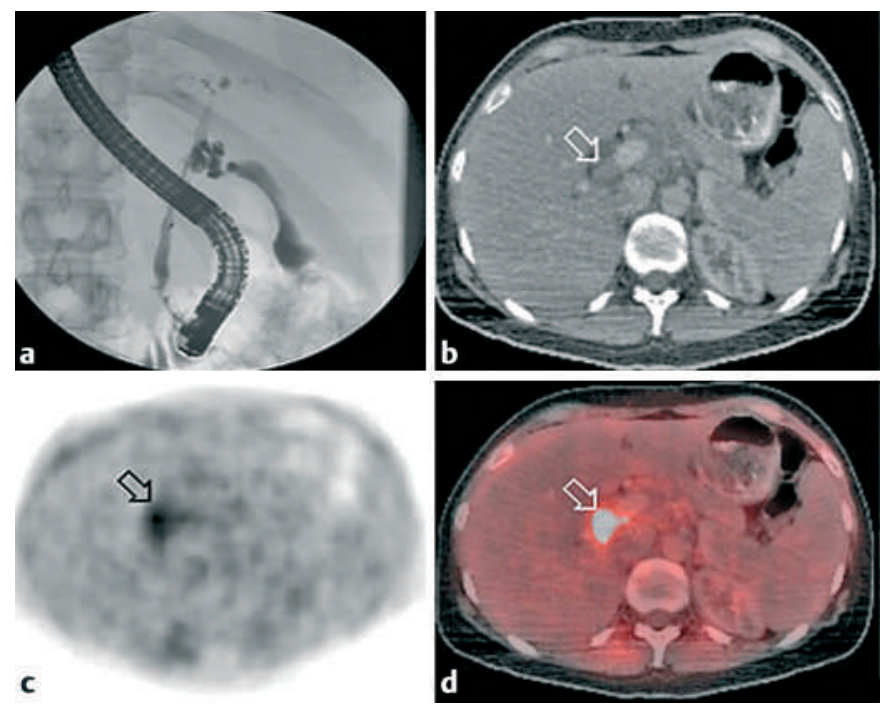

Figure 1 A 31-year-old woman was admitted to our hospital with a new episode of cholangitis, in the course of primary sclerosing cholangitis. Endoscopic and radiological evaluation suggested a bile duct carcinoma. a Diagnostic endoscopic retrograde cholangiography (ERC) showed typical findings of primary sclerosing cholangitis, as well as a suspicious lesion in the liver hilum. b Transverse computed tomography (CT) demonstrated a tumor lesion in the liver hilum. $\mathbf{c}$ Positron emission tomography (PET) images showed pathologically increased glucose metabolism in the upper abdomen. $\mathbf{d}$ This mapped accurately to the tumor lesion shown in $\mathbf{b}$, when fused PET and CT data were assessed. Exploratory laparotomy followed. Enlarged lymph nodes were identified in the liver hilum, that were causing dilatation of the common bile duct. Lymphadenectomy in the liver hilum and bile duct resection were performed, followed by hepaticojejunostomy with Roux-en-Y anastomosis. Surprisingly, histological investigation proved hepatobiliary sarcoidosis.
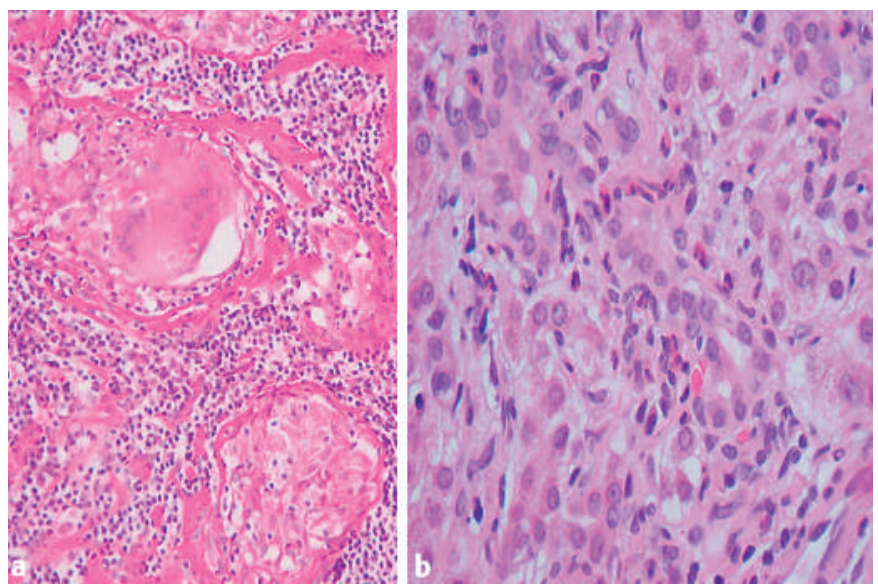

Figure 2 a On histological examination, the lymph nodes showed sarcoidal, noncaseating epitheloid granulomas. b Liver biopsy showed cholestasis, duct proliferation and granuloma formation, typical findings of granulomatous hepatitis. The masked clinical presentation of this case of Klatskin tumor-mimicking sarcoidosis reminds us of this rare cause of biliary tract obstruction. 